\title{
Changes in extracellular water and left ventricular mass in peritoneal dialysis patients
}

\author{
Theerasak Tangwonglert ${ }^{1}$, Andrew Davenport ${ }^{2}$ \\ ${ }^{1}$ Nephrology Division, Department of Medicine, Phramongkutklao Hospital, Bangkok, Thailand \\ ${ }^{2}$ UCL Department of Nephrology, Royal Free Hospital, University College London Medical School, London, United Kingdom
}

\begin{abstract}
Background: Increasing number of peritoneal dialysis (PD) patients are reported to have increased left ventricular hypertrophy (LVH), a major risk factor for cardiovascular mortality. We wished to determine which factors were most associated with changes in left ventricular mass index (LVMI).

Methods: We reviewed patient and treatment factors in prevalent PD patients with repeat echocardiograms 18 to 24 months apart, with corresponding bioimpedance measurements of extracellular water (ECW) and serum N-terminal pro-brain natriuretic peptide (NT-proBNP).

Results: We studied 60 patients (34 males, 35 with diabetes) who were treated with PD for a median of 14 months (2.5-26.3 months). All but one had LVH; on repeat echocardiography, there was no overall change in LVMI (106 [84-127] g/m² vs. 108 [91122] $\left.\mathrm{g} / \mathrm{m}^{2}\right)$ despite a loss of residual renal function. Left ventricular mass increased in $34(56.7 \%)$, and the percent change in LVMI was associated with percent change in NT-proBNP $(r=0.51, p=0.017)$ and ECW/height $(r=0.32, p=0.029)$, but not with ECW/total body water or changes in systolic or mean arterial pressure, urine output, 24-hour PD ultrafiltration, or net sodium balance. Only ECW/height remained independently associated with the percent change in LVMI in a multivariable model (odds ratio, 1.25; 95\% confidence interval, 1.08-1.36; $p=0.007$ ).

Conclusion: In this observational longitudinal report, a reduction in ECW/height was associated with regression of LVMI, whereas an increased ECW/height was associated with increased LVMI. As there was no corresponding association with systolic or mean arterial pressure, then volume expansion would appear to be a more significant factor in determining LVH than blood pressure.
\end{abstract}

Keywords: Hypertension, Hypertrophy, N-terminal pro-brain natriuretic peptide (1-76), Peritoneal dialysis; Water

\section{Introduction}

Although peritoneal dialysis (PD) does not lead to the repeated weekly volume and blood pressure changes associated with hemodialysis, cardiovascular mortality remains the most common cause of death [1]. A number of

Received: August 21, 2020; Revised: October 28, 2020; Accepted: November 8, 2020

Editor: Seung-Yeup Han, Keimyung University, Daegu, Republic of Korea

Correspondence: Andrew Davenport

UCL Department of Nephrology, Royal Free Hospital, University College London Medical School, Rowland Hill Street, London NW3 2PF, United Kingdom. E-mail: a.davenport@ucl.ac.uk

ORCID: https://orcid.org/0000-0002-4467-6833

Copyright (C) 2021 The Korean Society of Nephrology

(a) This is an Open Access article distributed under the terms of the Creative Commons Attribution Non-Commercial and No Derivatives License (http:// creativecommons.org/licenses/by-nc-nd/4.0/) which permits unrestricted non-commercial use, distribution of the material without any modifications, and reproduction in any medium, provided the original works properly cited. 
studies have observed that the majority of patients starting both hemodialysis and PD have left ventricular hypertrophy (LVH) $[2,3]$. Patients with chronic kidney disease have a number of potential risk factors for $\mathrm{LVH}$, as they are more likely to have hypertension, extracellular water (ECW) expansion, and anemia.

Previous observational cross-sectional studies have reported an association between LVH and increased mortality among PD patients [4]. Although many studies have reported on the results of a single echocardiogram in PD patients, very few studies have reviewed serial echocardiograms. Serial echocardiogram studies have reported varying results, with progression of LVH in one study of 12 patients, no change in a Chinese study of 40 patients, and a reduction in left ventricular mass (LVM) in a larger Korean study $[3,5,6]$. These studies were unable to address whether changes in LVM were related to changes in ECW, as LVM was reported to decrease even in patients who were persistently over-hydrated [6].

To determine whether LVM changes with ECW or other factors, we reviewed the results of prevalent PD patients with repeat echocardiograms and corresponding measurements of ECW, dialysis adequacy, and residual renal function.

\section{Methods}

We retrospectively reviewed data on adult PD patients who had visited a predialysis specialized chronic kidney disease clinic and then electively started PD and who had repeat outpatient echocardiograms 18 to 24 months after their first two-dimensional $\mathrm{M}$ mode transthoracic echocardiogram (Philips IE33; Philips Medical Systems, Eindhoven, the Netherlands). No patients had acute heart failure, acute coronary syndrome, or other acute cardiologic dysfunction at the time of imaging. Images were taken from the parasternal view with patients in the left decubitus position by trained and certified cardiology echocardiography technicians. LVM was calculated from the left ventricular posterior wall thickness, interventricular septal thickness, and left ventricular end-diastolic diameter using the Devereux formula [7]. To compare patients, LVH was adjusted for body size and then categorized by an LVM index (LVMI) of $>52 \mathrm{~g} / \mathrm{m}^{2}$ for males and $>47 \mathrm{~g} / \mathrm{m}^{2}$ for females, as recommended by the 2013 guidelines of the European Society of Hypertension (ESH) and the European
Society of Cardiology (ESC) [8]. Using the American Society of Echocardiography (ASE) recommendations to determine relative wall thickness (RWT), we then calculated the ratio of the intraventricular and posterior wall thickness to the left ventricular end-diastolic diameter, where ratios of $\leq 0.42$ and $>0.42$ describe eccentric and concentric LVH, respectively [9].

PD adequacy was calculated using standard methods from 24-hour urinary collections and samples taken from all spent dialysate bags and normalized protein nitrogen appearance was estimated using standard equations [10-12]. Blood glucose, glycated hemoglobin, C-reactive protein (CRP), N-terminal pro-brain natriuretic peptide (NTproBNP), and dialysate glucose were measured by standard methods (Roche Modular P analyzer; Roche Diagnostics Ltd., Burgess Hill, UK) [13]. Peritoneal membrane transport was calculated from a 4-hour peritoneal dialysate dwell and plasma creatinine concentrations using a standard 2.0-L 22.7 g/L glucose peritoneal dialysate (Baxter Health Care, Deerfield, MA, USA), with creatinine measured enzymatically (Roche Modular P analyzer) [14]. Patients were assigned as slow, medium, or fast transporter status according to European Dialysis and Transplant Best Practice guidelines [10].

To estimate combined peritoneal and urinary sodium losses, we used the inflow and drained volumes recorded by the automated PD (APD) cycler (Baxter Health Care), and measured sodium in the spent dialysate and 24-hour urine. For patients treated by continuous ambulatory PD (CAPD), patients and staff were instructed to allow 15 seconds for the flush-before-fill CAPD technique, and the median volume measured was $90 \mathrm{~mL}$, as such sodium in the spent CAPD dialysates were adjusted from an initial volume of $2.15 \mathrm{~L}$ in a fresh 2.0-L dialysate bag [15]. The amount of PD sodium removed was calculated as the difference between the amount of sodium instilled into the peritoneal cavity in 24 hours and that measured in the 24-hour drained effluent.

ECW, intracellular (ICW), and total body water (TBW) were measured using multifrequency bioelectrical impedance using a standardized protocol (InBody 720; InBody, Seoul, Korea), with peritoneal dialysate drained out and after voiding $[16,17]$. To make comparisons between patients, ECW was adjusted as ECW/TBW [18] and by height [19].

The Stoke-Davies grading system was used for patient 
comorbidity [20]. Other relevant information, including prescription of antihypertensive medications, was obtained from computerized hospital medical records.

\section{Ethics}

Our retrospective audit complied with the United Kingdom National Health Service Health Research Authority guidelines for clinical audit and service development (https://www. hra.nhs.uk) and was registered with the UCL Department of Nephrology Royal Free Hospital. All patient data were anonymized in keeping with the Helsinki accord.

\section{Statistical analysis}

Statistical analysis was performed by using D'Agostino Pearson testing for analysis of normality and groups were compared using t tests and Mann-Whitney U tests, chi-square tests, and paired $\mathrm{t}$ tests, Wilcoxon rank-sum pair testing with appropriate correction for multiple testing by Bonferroni and Tukey post hoc testing, and for small numbers. We used Spearman univariate correlation and standard statistical programs (IBM SPSS, version 24.0; IBM, Armonk, NY, USA and GraphPad Prism, version 9.0; GraphPad Software, San Diego, USA). A step-backward multivariable logistic regression model was created comparing patients who had an increase in LVM compared to those with a fall in LVM, using all variables with a univariate association of $\mathrm{p}<0.1$, and were then eliminated if not statistically significant or did not improve the model fit. Data are presented as either the mean \pm standard deviation, median (interquartile range), mean with $95 \%$ confidence interval of agreement, or as a percentage.

\section{Results}

We reviewed the records of 60 prevalent PD patients who had repeat echocardiograms 18 to 24 months apart, 34 (56.7\%) were males and 35 (58.3\%) had diabetes (Table 1 ). Nine patients $(15.0 \%)$ had a prior medical history of an acute coronary syndrome, nine $(15.0 \%)$ with coronary artery stenting, and four $(6.7 \%)$ had undergone coronary artery bypass surgery. All male patients $(100 \%)$ had LVH on both echocardiograms, $98.3 \%$ of the female patients had LVH at the time of the first echocardiogram, and $100 \%$ of the
Table 1. Patient demographics at the time of the first and second echocardiogram

\begin{tabular}{|c|c|c|}
\hline \multirow{2}{*}{ Variable } & \multicolumn{2}{|c|}{ Echocardiogram } \\
\hline & First & Second \\
\hline Age (yr) & $65.2 \pm 13.9$ & $66.9 \pm 13.9 * * *$ \\
\hline Weight (kg) & $69.8 \pm 14.0$ & $68.9 \pm 13.1$ \\
\hline Comorbidity grade $^{a}$ & I (0-II) & $I(0-I I)$ \\
\hline CAPD & $22(36.7)$ & $21(35.0)$ \\
\hline APD & $7(11.7)$ & $6(10.0)$ \\
\hline CCPD & $30(50.0)$ & 32 (53.3) \\
\hline Using icodextrin & $43(71.7)$ & $52(86.7)^{*}$ \\
\hline Using $22.7 \mathrm{~g} / \mathrm{L}$ glucose & $31(51.7)$ & $39(65.0)$ \\
\hline \multicolumn{3}{|l|}{ Weekly Kt/V } \\
\hline For urine & $0.99(0.54-0.78)$ & $0.30(0-1.05) * * *$ \\
\hline For perzneal dialysis & 1.54 (1.38-1.94) & $1.41(1.11-1.78)$ \\
\hline For total & $2.31(1.75-3.03)$ & $2.04(1.57-2.55)^{*}$ \\
\hline $\begin{array}{l}\text { Peritoneal ultrafiltration } \\
(\mathrm{mL} / \text { day })\end{array}$ & $594(252-881)$ & $826(507-1,316)^{*}$ \\
\hline nPNA (g/kg/day) & $0.88(0.75-1.09)$ & $0.80(0.70-0.99)^{* *}$ \\
\hline $\begin{array}{l}\text { Sodium balance } \\
\text { (mmol/day) }\end{array}$ & $-119(-159$ to -68$)$ & $-105(-154$ to -75$)$ \\
\hline $\begin{array}{l}\text { 4-hr dialysate/plasma } \\
\text { creatinine }\end{array}$ & $0.69 \pm 0.15$ & $0.69 \pm 0.17$ \\
\hline LV mass index $\left(\mathrm{kg} / \mathrm{m}^{2}\right)$ & $106(84-127)$ & $108(91-122)$ \\
\hline LV ejection fraction (\%) & $57(47-59)$ & $57(47-58)$ \\
\hline $\begin{array}{l}\text { Relative wall thickness } \\
\text { ratio }\end{array}$ & $0.48 \pm 0.11$ & $0.50 \pm 0.12$ \\
\hline NT-proBNP (pmol/L) & $2,216(732-8,926)$ & $5,201(1,505-17,481)^{*}$ \\
\hline ECW/TBW & $0.395 \pm 0.013$ & $0.397 \pm 0.011$ \\
\hline ECW/height (L/m) & $8.71 \pm 1.66$ & $8.15 \pm 1.35 *$ \\
\hline Hemoglobin (g/L) & $94.2 \pm 12.7$ & $90.0 \pm 12.9$ \\
\hline Albumin (g/L) & $39.1 \pm 3.4$ & $36.8 \pm 4.4$ \\
\hline $\begin{array}{l}\text { C-reactive protein } \\
(\mathrm{mg} / \mathrm{L})\end{array}$ & $3.0(1.0-7.5)$ & $5.0(2.0-8.8)^{*}$ \\
\hline \multicolumn{3}{|l|}{ Blood pressure (mmHg) } \\
\hline Systolic & $139.3 \pm 28.5$ & $143.5 \pm 27.8$ \\
\hline Diastolic & $79.1 \pm 16.7$ & $79.9 \pm 15.9$ \\
\hline $\begin{array}{l}\text { Antihypertensive } \\
\text { medication }^{c}\end{array}$ & $1(0-2)$ & $1(0-2)$ \\
\hline
\end{tabular}

Data are expressed as mean \pm standard deviation, median (interquartile range), or number (\%).

APD, automated peritoneal dialysis; CAPD, continuous ambulatory peritoneal dialysis; CCPD, continuous cycling peritoneal dialysis; ECW, extracellular water; LV, left ventricular; nPNA, normalized protein nitrogen appearance; NT-proBNP, N-terminal pro-brain natriuretic peptide; TBW, total body water.

${ }^{a}$ According to Stoke-Davies classification. ${ }^{b}$ Net difference between peritoneal and urinary sodium loss. ${ }^{\circ}$ Number of different classes of antihypertensive medications.

$* p<0.05, * * p<0.01$, and $* * * p<0.001$ vs. first echocardiogram. 
females had LVH on the repeat echocardiogram. Volume overload is thought to be major factor in the development of eccentric LVH, and blood pressure the major factor for concentric hypertrophy. Although there is some debate as to the cut-off defining eccentric and concentric hypertrophy, use of the ASE recommendations revealed that $55.0 \%$ of the patients had concentric LVH [9].

Although there was no overall change in LVM or LVMI for the cohort, LVM increased in 34 patients (56.7\%) and fell in 26 (43.3\%). As such, we compared the characteristics of these two groups (Table 2). The median time between the bioimpedance and the first echocardiogram and was 1 month (1-3 months), and for the second echocardiogram was 2 months (1-2.3 months). There were no differences in age, comorbidity, or months of PD treatment at the time of the initial or follow-up echocardiogram between the groups (increased LVM vs. decreased LVM: acute coronary artery, syndrome $20.6 \%$ vs. $6.9 \%$; coronary artery stenting, $17.7 \%$ vs. $13.8 \%$; coronary artery bypass grafting, $5.9 \%$ vs. $6.9 \%$; all $p$ $>0.05)$. Left ventricular ejection fractions did not differ, and the percentage of patients with concentric hypertrophy was marginally but not significantly greater for those patients with a fall in LVM (88.5\% vs. $73.6 \%$ ). Similarly, there were no differences in PD adequacy, ultrafiltration, or net peritoneal sodium balance. However, no patients in the group with a fall in LVMI were initially treated by APD. Although ECW/ TBW, ECW/height, and NT-proBNP at the time of the first echocardiogram were similar, those patients who had a subsequent decrease in LVMI initially had a greater LVMI (Fig. 1), despite similar clinical blood pressure recordings and prescription of antihypertensive medications. Initially, 18 patients were taking $\beta$-blockers, 16 were taking calcium channel blockers/ $\alpha$-blockers, nine were taking reninangiotensin blockers/receptor blockers, and 41 were taking

Table 2. Patient demographics and peritoneal dialysis prescriptions according to change in LV mass

\begin{tabular}{|c|c|c|c|c|}
\hline Variable & Decreased, first Echo & LV mass, second Echo & Increased, first Echo & LV mass, second Echo \\
\hline Age (yr) & $66.0 \pm 12.0$ & $68.2 \pm 11.9$ & $64.1 \pm 15.3$ & $66.0 \pm 14.5$ \\
\hline Weight (kg) & $70.3 \pm 12.8$ & $68.0 \pm 10.2$ & $69.4 \pm 15.0$ & $69.4 \pm 15.2$ \\
\hline Peritoneal dialysis (mo) & $13.5(1-24)$ & $30.0(20-44)$ & $18.0(2-27)$ & $33.5(23-52)$ \\
\hline CAPD/APD/CCPD & $34.6 / 0 / 65.3 *$ & $38.2 / 20.6 / 38.2$ & $34.6 / 3.8 / 61.5$ & $35.3 / 14.7 / 50.0$ \\
\hline \multicolumn{5}{|l|}{ Weekly Kt/V } \\
\hline For urine & $0.86(0.40-1.75)$ & $0.32(0-1.20)$ & $1.28(0.95-1.51)$ & $0.23(0.05-0.86)$ \\
\hline For peritoneal dialysis & $1.47(1.01-1.65)$ & $1.43(1.21-1.75)$ & $1.28(0.95-1.51)$ & $1.37(0.97-1.79)$ \\
\hline Peritoneal ultrafiltration ( $\mathrm{mL} /$ day) & $534(221-900)$ & $977(412-1,200)$ & $565(352-866)$ & $722(546-1,384)$ \\
\hline Sodium balance ${ }^{a}(\mathrm{mmol} /$ day $)$ & $-107(-160$ to -65$)$ & $-107(-175$ to -82$)$ & $-127(-155$ to -84$)$ & $-105(-154$ to -78$)$ \\
\hline nPNA (g/kg/day) & $0.94 \pm 0.28$ & $0.84 \pm 0.26$ & $0.95 \pm 0.22$ & $0.86 \pm 0.19$ \\
\hline 4-hr dialysate/plasma creatinine & $0.72 \pm 0.14$ & $0.68 \pm 0.20$ & $0.64 \pm 0.14$ & $0.71 \pm 0.14$ \\
\hline Albumin (g/L) & $37.9 \pm 3.4 *$ & $36.7 \pm 4.0$ & $40.1 \pm 3.1$ & $36.9 \pm 4.7$ \\
\hline C-reacitve protein (mg/L) & $4.0(2.0-12.0)^{*}$ & $6.5(2.0-11.0)$ & $2.0(0.5-11.0)$ & $3.5(2.0-8.0)$ \\
\hline NT-proBNP (pmol/L) & $3,814(1,624-14,470)$ & $2,134(1,446-7,298)$ & $1,395(516-6,106)^{*}$ & $7,265(2,263-22,411)$ \\
\hline ECW/TBW & $0.394 \pm 0.013$ & $0.397 \pm 0.009$ & $0.396 \pm 0.013$ & $0.397 \pm 0.013$ \\
\hline ECW/height $(\mathrm{L} / \mathrm{m})$ & $8.7(7.4-9.3)$ & $7.7(7.0-8.7)$ & $8.6(7.4-9.8)$ & $8.2(7.4-9.1)$ \\
\hline \multicolumn{5}{|l|}{ Blood pressure (mmHg) } \\
\hline Systolic & $144 \pm 30$ & $141 \pm 27$ & $136 \pm 28$ & $146 \pm 28$ \\
\hline Diastolic & $82 \pm 19$ & $79 \pm 15$ & $77 \pm 15$ & $81 \pm 17$ \\
\hline Antihypertensive medication $^{\mathrm{b}}$ & $1(0-1)$ & $1(0-1)$ & $1(0-2)$ & $1(0-2)$ \\
\hline LV ejection fraction (\%) & $57(52-58)$ & $58(48-58)$ & $57(47-60)$ & $57(47-60)$ \\
\hline Relative wall thickness ratio & $0.50 \pm 0.12$ & $0.53 \pm 0.14$ & $0.48 \pm 0.11$ & $0.48 \pm 0.09$ \\
\hline
\end{tabular}

Data are expressed as mean \pm standard deviation, median (interquartile range), or number (\%).

APD, automated peritoneal dialysis; CAPD, continuous ambulatory peritoneal dialysis; CCPD, continuous cycling peritoneal dialysis; Echo, echocardiogram; ECW, extracellular water; LV, left ventricular; nPNA, normalized protein nitrogen appearance; NT-proBNP, N-terminal pro-brain natriuretic peptide; TBW, total body water.

${ }^{a}$ Net difference between peritoneal and urinary sodium loss. ${ }^{b}$ Number of different classes of antihypertensive medications prescribed.

$*_{p}<0.05$ vs. increased LV mass group. 
loop diuretics. At the time of the second echocardiogram, 23 patients were on $\beta$-blockers, 16 were on calcium channel blockers/ $\alpha$-blockers, 12 were on renin-angiotensin blockers/ receptor blockers, and 43 were on loop diuretics. There were no differences between the groups in terms of the proportion of prescribed renin-angiotensin inhibitors/

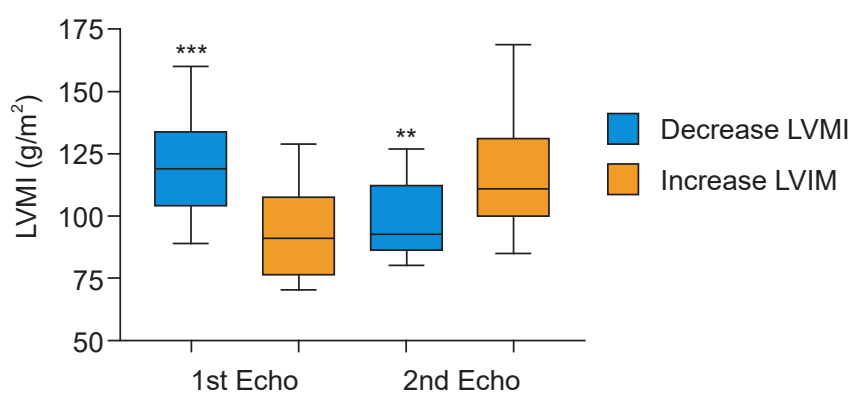

Figure 1. Left ventricular mass index (LVMI) obtained by transthoracic two-dimensional echocardiography (Echo). Medians, interquartile ranges, and 90\% confidence intervals are indicated.

$* * p<0.01$ and $* * * p<0.001$ vs. the group with increased LVMI. receptor blockers, or $\beta$ - or calcium channel blockers.

At the time of the repeat echocardiogram, NT-proBNP was greater in the group that had an increase in LVMI (Table 2), as were the changes in NT-proBNP and ECW/height (Table 3). The changes in systolic blood pressure and mean arterial pressure were greater for the group with an increase in LVM, but after adjusting for multiple statistical testing, the difference was no longer statistically significant. There was no statistical difference in the changes made in the prescription of antihypertensive medications. Although patients who had a reduction in LVM had lost weight (unadjusted $\mathrm{p}=0.025$ ) (Table 2), there were no significant changes in body weights between the groups (Table 3 ).

On univariate analysis, the percent change in LVMI was associated with the percent change in NT-proBNP $(r=0.51$, $\mathrm{p}=0.017)$ and percent change in ECW/height $(\mathrm{r}=0.32, \mathrm{p}$ $=0.029$ ), but not with ECW/TBW or changes in systolic or mean arterial pressure, urine output, 24-hour ultrafiltration, weight, or net sodium balance. As the change in LVMI was nonparametric, with both positive and negative values, we

Table 3. Patients divided according to change in LVM

\begin{tabular}{|c|c|c|}
\hline Change & Decreased LVM & Increased LVM \\
\hline Weight (kg) & $-1.8(-5.4$ to 1.1$)$ & $-0.6(-3.9$ to 2.8$)$ \\
\hline Reduction in urine Kt/V & $0.29(0-0.59)$ & $0.36(0-0.88)$ \\
\hline Reduction in total Kt/V & $0.19(-0.03$ to 0.48$)$ & $0.32(-0.83$ to 0.08$)$ \\
\hline Peritoneal ultrafiltration (mL/day) & $484(0-635)$ & $130(0-320)$ \\
\hline Reduction in nPNA (g/kg/day) & $0.05(-0.19$ to 0.02$)$ & $0.08(-0.16$ to 0.01$)$ \\
\hline 4-hr dialysate/plasma creatinine & $0.03(0.07-0.1)$ & $0.04(-0.1-0)$ \\
\hline NT-proBNP (pmol/L) & $-8.5(-4,739$ to 440$)$ & $206(-560 \text { to } 13,641)^{* *}$ \\
\hline ECW/TBW & $0.004(-0.002$ to 0.008$)$ & 0.006 (-0.001 to 0.009) \\
\hline ECW/height( L/m) & $-0.50(-0.74$ to 0.12$)$ & $0.21(-0.10$ to 0.50$)$ * \\
\hline Albumin (g/L) & $0(0-1.0)$ & $0(0-0)$ \\
\hline Diastolic & $-1.5(-16.0$ to 5.0$)$ & $3.0(-8.5$ to 17.0$)$ \\
\hline Mean arterial pressure (mmHg) & $-3.5(-17.0$ to 5.7$)$ & $6.3(-6.3 \text { to } 20.3)^{*}$ \\
\hline \multicolumn{3}{|l|}{ Antihypertensive medication ${ }^{\mathrm{b}}(\%)$} \\
\hline Increase & $6(23.1)$ & $7(20.6)$ \\
\hline Reduction & $2(7.7)$ & $9(26.5)$ \\
\hline
\end{tabular}

Data are expressed as median (interquartile range) or number (\%).

LVM, left ventricular mass; PD, peritoneal dialysis; nPNA, normalized protein nitrogen appearance; ECW, extracellular water; NT-proBNP, N-terminal probrain natriuretic peptide; TBW, total body water.

${ }^{a}$ Net difference between peritoneal and urinary sodium loss. ${ }^{b}$ Number of different classes of antihypertensive medications prescribed.

$* p<0.05$ and $* * p<0.01$ vs. the decreased LVM group. 
constructed a binary logistic step-backward multivariable model, including all variables with $\mathrm{p}<0.1(22.7 \mathrm{~g} / \mathrm{L}$ dialysate, total weekly Kt/V, 24-hour PD ultrafiltration volume, percent change ECW/height, percent change in NT-proBNP, serum albumin, and serum CRP). Only ECW/height remained independently associated with the percent change in LVMI (odds ratio, 1.25; 95\% confidence interval, 1.08-1.36; $\mathrm{p}=$ 0.007 , Nagelkerke ${ }^{2}=0.54$ ).

\section{Discussion}

Several observational studies found that the majority of PD patients have increased ECW, yet without overt clinical signs of volume overload [21,22]. Interventional studies in hemodialysis patients, driven by bioimpedance to reduce ECW, have reported a reduction in both blood pressure and LVM [23], but similar studies in PD patients have failed to demonstrate a major change in blood pressure control $[6,24]$. This may be due to the more regular assessment of hemodialysis patient target weights, the effect of residual renal function in PD patients, and differences in sodium removal between the two therapies.

To reduce the confounding effects of predialysis care, we reviewed echocardiograms in prevalent PD patients who had electively started PD following clinical care in a specialized chronic kidney disease clinic designed to prepare patients for dialysis. Despite specialized care, all but one patient had LVH by echocardiographic criteria [8], consistent with previous reports of an increased prevalence of LVH in PD patients [3-5].

We noted that, whereas there was no overall change in blood pressure control or LVM, LVM increased in the majority of our patients. To determine which factors contributed to changes in LVM, we compared changes in PD prescriptions, PD adequacy, blood pressure measurements, and assessment of volume status in patients who underwent repeat outpatient echocardiogram 18 to 24 months after the first echocardiogram. As expected with time, residual renal function declined, leading to a fall in total weekly $\mathrm{Kt} / \mathrm{V}$. To compensate for the loss in residual renal function, 24-hour PD ultrafiltration volumes increased, along with an increase in the prescription of icodextrin dialysates. In terms of ECW volume, whereas there were no changes in ECW/TBW ratios, ECW/height declined overall, with a greater change in patients with a reduction in LVM. The ratio of ECW/TBW not only reflects ECW but is also influenced by loss of ICW [18], so it can be affected by changes in body composition and inflammation [25]. NT-proBNP concentrations increased over time, and although often used as a biomarker of intravascular volume [26], NT-proBNP can increase with a fall in residual renal function, valvular heart disease, cardiac arrhythmias, and inflammation [13,27]. However, brain natriuretic peptide has not been shown to be affected by $\mathrm{LVH}$ in patients with essential hypertension [28]. We observed that NT-proBNP increased in patients who had an increase in LVM, but not in patients who had a fall in LVM.

Comparing patients who had an increase or decrease in LVM over time, we found no differences in residual renal function, PD adequacy, PD ultrafiltration volumes, peritoneal transporter status, use of icodextrin, or hypertonic glucose dialysates. Although the change in weight between groups was not significant, there was an overall fall in body weight for patients with a reduction in LVM, and there was no correlation between weight loss and change in LVM. More patients with an increase in LVM were initially treated by APD cyclers, which have been reported to result in lower PD sodium removal compared to CAPD and continuous cycling peritoneal dialysis [15]. However, we found no difference in combined urinary and peritoneal sodium removal, but this estimation of sodium balance excludes dietary sodium, as we were unable to accurately record dietary sodium intake over time. Although absolute systolic and mean arterial pressures were not different, the change in blood pressure was greater in the group with an increase in LVM, as were the changes in NT-proBNP and ECW adjusted for height [19], and both were associated with the change in LVM. However, on multivariable testing, only the ratio of ECW/height remained independently associated with the change in LVM, suggesting that volume status, in particular ECW excess, is associated with increasing LVM. Our data is supported in reverse by one previous study which reported that a reduction in ECW was associated with a reduction in LVM, but equally LVM was also reduced in their cohort of patients who remained volume overloaded [6].

Longitudinal studies in PD patients have several confounders, due to patient drop-out, failure to follow techniques, peritonitis, transplantation, and mortality. As such, the number of patients with repeated measurements generally leads to reports from small observational studies. Thus, we report on a small cohort of 60 PD patients studied 
in a single center, but this compares favorably to two earlier studies of 12 and 40 patients, respectively [3,5]. There has been debate regarding how to best adjust LVM to compare differences between patients [29]. We compared changes in LVM and LVMI in paired echocardiograms as advised by the European Society of Cardiology [8]. As remodeling of the left ventricular takes many months, we chose to compare echocardiograms 18 to 24 months apart, and as such accepted a short interval between echocardiograms and bioimpedance measurements. The majority of our patients had concentric LVH based on RWT criteria, and although there has been debate as to what RWT cut-off point should be used to categorize patients as having concentric and eccentric LVH, specifically 0.42 or 0.45 , the percentage of patients with concentric hypertrophy only changed from $56.7 \%$ to $55 \%$ [9]. The greater concern is whether a single cutoff point can reliably separate concentric and eccentric LVH. We used bioimpedance to assess ECW, and as some studies have suggested that measurement of ECW can be affected by the presence of peritoneal dialysate, we took measurements after dialysate had been drained [17].

In hemodialysis, volume excess is a major risk factor for death, and in particular cardiovascular death. Reports in hemodialysis patients have linked ECW excess with LVH [1]. Although observational studies in PD patients have reported a high prevalence of LVH in PD patients [21], with an associated increased risk of cardiovascular mortality [2], these cross-sectional, single time point observational studies have been unable to establish the major causes of LVH. As residual renal function is important for PD patients, there have been concerns as to whether reducing ECW may lead to a premature loss of residual renal function, although studies have not supported this [30]. Our longitudinal observational work suggests a role for ECW excess as a driving force for increasing LVM, rather than blood pressure control.

Although there remains debate about the importance of ECW overload in PD patients, with meta-analyses failing to demonstrate a definitive association between PD technique failure and mortality [31], our study would suggest that greater attention to ECW could potentially prevent progression of LVM in PD patients.

\section{Conflicts of interest}

All authors have no conflicts of interest to declare.

\section{Authors' contributions}

Conceptualization, Project administration, Visualization: AD Data curation, Formal analysis, Investigation, Methodology: TW

Writing-original draft: $\mathrm{AD}$

Writing-review \& editing: TW, AD

All authors read and approved the final manuscript.

\section{ORCID}

Theerasak Tangwonglert, https://orcid.org/0000-0001-6475-0407

Andrew Davenport, https://orcid.org/0000-0002-4467-6833

\section{References}

1. Banshodani M, Kawanishi H, Moriishi M, Shintaku S, Tsuchiya S. Association between dialysis modality and cardiovascular diseases: a comparison between peritoneal dialysis and hemodialysis. Blood Purif 2020;49:302-309.

2. Foley RN, Parfrey PS, Harnett JD, et al. Clinical and echocardiographic disease in patients starting end-stage renal disease therapy. Kidney Int 1995;47:186-192.

3. Hüting J, Alpert MA. Progression of left ventricular hypertrophy in end-stage renal disease treated by continuous ambulatory peritoneal dialysis depends on hypertension and hypercirculation. Clin Cardiol 1992;15:190-196.

4. Wang AY, Wang M, Woo J, et al. Inflammation, residual kidney function, and cardiac hypertrophy are interrelated and combine adversely to enhance mortality and cardiovascular death risk of peritoneal dialysis patients. J Am Soc Nephrol 2004;15:21862194.

5. Shi Q, Zhu J, Feng S, Shen H, Chen J, Song K. Nonparallel progression of left ventricular structure and function in longterm peritoneal dialysis patients. Cardiorenal Med 2017;7:198206.

6. Hong YA, Yoon HE, Choi BS, et al. The effect of strict volume control assessed by repeated bioimpedance spectroscopy on cardiac function in peritoneal dialysis patients. Sci Rep 2019; 9:17679.

7. Devereux RB, Alonso DR, Lutas EM, et al. Echocardiographic assessment of left ventricular hypertrophy: comparison to necropsy findings. Am J Cardiol 1986;57:450-458.

8. Mancia G, Fagard R, Narkiewicz K, et al. 2013 ESH/ESC guidelines for the management of arterial hypertension: the 
task force for the management of arterial hypertension of the European Society of Hypertension (ESH) and of the European Society of Cardiology (ESC). J Hypertens 2013;31:1281-1357.

9. Sahn DJ, DeMaria A, Kisslo J, Weyman A. Recommendations regarding quantitation in M-mode echocardiography: results of a survey of echocardiographic measurements. Circulation 1978;58:1072-1083.

10. van Biesen W, Heimburger O, Krediet R, et al. Evaluation of peritoneal membrane characteristics: clinical advice for prescription management by the ERBP working group. Nephrol Dial Transplant 2010;25:2052-2062.

11. Dombros N, Dratwa M, Feriani M, et al. European best practice guidelines for peritoneal dialysis. 7 Adequacy of peritoneal dialysis. Nephrol Dial Transplant 2005;20 Suppl 9:ix24-ix27.

12. Vongsanim S, Salame C, Eaton S, Grimble G, Davenport A. Differences between measured total nitrogen losses in spent peritoneal dialysate effluent and estimated nitrogen losses.J Ren Nutr 2019;29:243-247.

13. Papakrivopoulou E, Lillywhite S, Davenport A. Is N-terminal probrain-type natriuretic peptide a clinically useful biomarker of volume overload in peritoneal dialysis patients? Nephrol Dial Transplant 2012;27:396-401.

14. Persaud J, Thomas M, Davenport A. Indirect ion selective electrode methods potentially overestimate peritoneal dialysate sodium losses. Ther Apher Dial 2014;18:321-325.

15. Maharjan SR, Davenport A. Comparison of sodium removal in peritoneal dialysis patients treated by continuous ambulatory and automated peritoneal dialysis. J Nephrol 2019;32:1011-1019.

16. Fürstenberg A, Davenport A. Comparison of multifrequency bioelectrical impedance analysis and dual-energy X-ray absorptiometry assessments in outpatient hemodialysis patients. Am J Kidney Dis 2011;57:123-129.

17. Davenport A. Effect of intra-abdominal dialysate on bioimpedancederived fluid volume status and body composition measurements in peritoneal dialysis patients. Perit Dial Int 2013;33:578-579.

18. Davies SJ, Davenport A. The role of bioimpedance and biomarkers in helping to aid clinical decision-making of volume assessments in dialysis patients. Kidney Int 2014;86:489-496.

19. van de Kerkhof J, Hermans M, Beerenhout C, Konings C, van der Sande FM, Kooman JP. Reference values for multifrequency bioimpedance analysis in dialysis patients. Blood Purif 2004;22: 301-306.

20. Davies SJ. Assessment of comorbidity in peritoneal dialysis patients. Contrib Nephrol 2003;(140):98-103.

21. Papakrivopoulou E, Booth J, Pinney J, Davenport A. Comparison of volume status in asymptomatic haemodialysis and peritoneal dialysis outpatients. Nephron Extra 2012;2:48-54.

22. Enia G, Mallamaci F, Benedetto FA, et al. Long-term CAPD patients are volume expanded and display more severe left ventricular hypertrophy than haemodialysis patients. Nephrol Dial Transplant 2001;16:1459-1464.

23. Hur E, Usta M, Toz H, et al. Effect of fluid management guided by bioimpedance spectroscopy on cardiovascular parameters in hemodialysis patients: a randomized controlled trial. $\mathrm{Am} \mathrm{J}$ Kidney Dis 2013;61:957-965.

24. Oh KH, Baek SH, Joo KW, et al. Does routine bioimpedanceguided fluid management provide additional benefit to nonanuric peritoneal dialysis patients?: results from COMPASS clinical trial. Perit Dial Int 2018;38:131-138.

25. Yoowannakul S, Tangvoraphonkchai K, Davenport A. The prevalence of muscle wasting (sarcopenia) in peritoneal dialysis patients varies with ethnicity due to differences in muscle mass measured by bioimpedance. Eur J Clin Nutr 2018;72:381-387.

26. Arrigo M, Von Moos S, Gerritsen K, et al. Soluble CD146 and B-type natriuretic peptide dissect overhydration into functional components of prognostic relevance in haemodialysis patients. Nephrol Dial Transplant 2018;33:2035-2042.

27. Fukuda N, Shinohara H, Sakabe K, Nada T, Tamura Y. Plasma levels of brain natriuretic peptide in various forms of obstruction to the left ventricular outflow tract. J Heart Valve Dis 2003;12:333340.

28. Nishikimi T, Yoshihara F, Morimoto A, et al. Relationship between left ventricular geometry and natriuretic peptide levels in essential hypertension. Hypertension 1996;28:22-30.

29. Foppa M, Duncan BB, Rohde LE. Echocardiography-based left ventricular mass estimation: how should we define hypertrophy? Cardiovasc Ultrasound 2005;3:17.

30. McCafferty K, Fan S, Davenport A. Extracellular volume expansion, measured by multifrequency bioimpedance, does not help preserve residual renal function in peritoneal dialysis patients. Kidney Int 2014;85:151-157.

31. Shu Y, Liu J, Zeng X, et al. The effect of overhydration on mortality and technique failure among peritoneal dialysis patients: a systematic review and meta-analysis. Blood Purif 2018;46:350358. 